\title{
Fast Electrical Control of a Quantum Dot Strongly Coupled to a Photonic-Crystal Cavity
}

\author{
Andrei Faraon, ${ }^{1,2, *}$ Arka Majumdar, ${ }^{1}$ Hyochul Kim, ${ }^{3}$ Pierre Petroff, ${ }^{3}$ and Jelena Vučković ${ }^{1}$ \\ ${ }^{1}$ E. L. Ginzton Laboratory, Stanford University, Stanford, California 94305, USA \\ ${ }^{2}$ Hewlett-Packard Laboratories, 1501 Page Mill Road, Palo Alto, California 94304, USA \\ ${ }^{3}$ Department of Electrical and Computer Engineering, University of California, Santa Barbara, California 93106, USA
}

(Received 4 June 2009; published 27 January 2010)

\begin{abstract}
The resonance frequency of an InAs quantum dot strongly coupled to a GaAs photonic-crystal cavity was electrically controlled via the quadratic quantum confined Stark effect. Stark shifts up to $0.3 \mathrm{meV}$ were achieved using a lateral Schottky electrode that created a local depletion region at the location of the quantum dot. We report switching of a probe laser coherently coupled to the cavity up to speeds as high as $150 \mathrm{MHz}$, limited by the $R C$ constant of the transmission line. The coupling strength $g$ and the magnitude of the Stark shift with electric field were investigated while coherently probing the system.
\end{abstract}

PACS numbers: 78.67.Hc, 42.50.Ct, 42.50.Pq, 78.20.Jq

Quantum dots (QDs) and photonic crystals (PCs) can be integrated into one of the most promising platforms for optical information processing at the micro- and nanoscale [1-3]. We have already shown that the transmission function of photonic-crystal cavities integrated in basic photonic-crystal networks can be controlled using coupled quantum dots [2,4]. In order to achieve ultrafast speeds of operation, the QDs should be controlled either optically $[5,6]$ or electrically. Here we show fast electrical control of strongly coupled QDs in PC cavities [7,8] using the quantum confined Stark effect (QCSE) [9] with lateral electrodes. The lateral electrode configuration provides a simple yet very effective method to control QDs in cavities that could be easily integrated in optical networks with higher degrees of complexity. The device was used to electrically modulate a laser beam resonantly coupled to the cavity, thus demonstrating an electro-optic modulator based on a single quantum emitter. The effective volume of the modulator could be as low as the optical mode volume of the cavity, which should enable switching energies as low as $1 \mathrm{fJ}$, which could push the boundary of energy consumption for the future generation of electro-optic switches [10]. This device operates at the fundamental limit of light-matter interaction, and it adds an essential component to the toolbox of quantum optical technologies.

The device consists of an InAs quantum dot coupled to a linear three hole defect photonic-crystal cavity [11] fabricated in a 160-nm-thick GaAs membrane [Fig. 1(a)]. The electrical control was achieved by applying a lateral electric field across the quantum dot and thus shifting its resonant frequency via QCSE $[12,13]$. The field was created in the depletion layer of a Schottky contact $(20 \mathrm{~nm}$ $\mathrm{Cr} / 25 \mathrm{~nm} \mathrm{Au}$ on GaAs) deposited in the vicinity of the quantum dot [14]. A scanning electron microscope image of the photonic-crystal resonator integrated with the laterally positioned electrode is shown in Fig. 1(a). Another Schottky contact, located on the surface of the chip a few hundred microns away from the photonic crystal, was used to set the ground potential.

One challenge in designing the device is the small extent of the depletion layer in the vicinity of the Schottky contact. For typical undoped GaAs grown by molecular beam epitaxy, there is still a dopant concentration of $\sim 10^{16} / \mathrm{cm}^{3}$

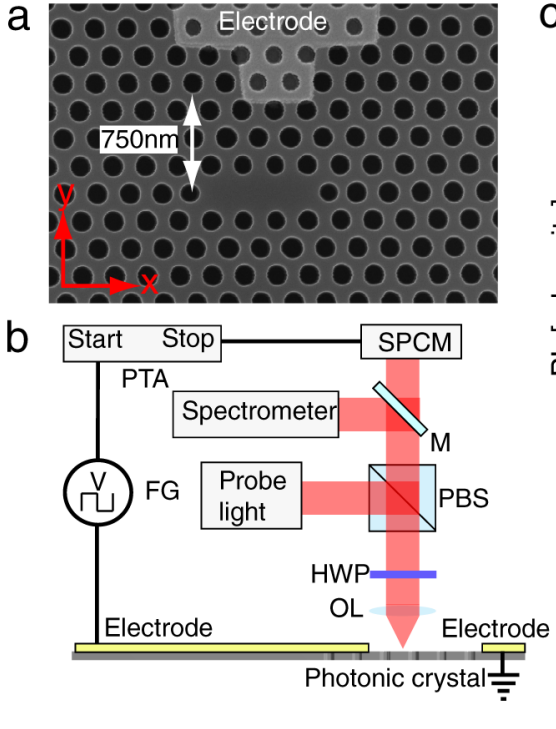

FIG. 1 (color online). (a) Scanning electron microscope image of the photonic-crystal cavity and the metallic electrode placed within $1 \mu \mathrm{m}$ from the center of the cavity. (b) Schematic representation of the experimental setup (not drawn to scale). A cross-polarized confocal microscope setup composed of a polarizing beam splitter (PBS), half-wave plate (HWP), and objective lens (OL) was used for photoluminescence and reflectivity measurements. The voltage on the chip was controlled using a function generator $(\mathrm{FG})$, and the output signal was detected using a single-photon counting module (SPCM). A flip mirror (M) switched the signal from spectrometer to SPCM. The timedomain measurements were performed using a picosecond time analyzer (PTA). (c) Photoluminescence spectra taken for different cavity/QD detunings by increasing the temperature of the sample (indicated for each spectrum). The avoided crossing of the polaritons [marked by the (red) lines as a guide to the eye] indicates that QD1 is strongly coupled. 
that limits the size of the depletion layer to a few microns for a $10 \mathrm{~V}$ bias voltage. This requires the contact to be brought into a proximity of a few microns from the quantum dot embedded in the photonic-crystal cavity. Since metals introduce high optical losses, the device was designed such that the metal electrode, located within $\sim 1 \mu \mathrm{m}$ from the center of the resonator, had a minimum overlap with the optical mode. The fundamental mode of the resonator extends mainly in a direction that makes an angle of $\sim 30^{\circ}$ with the cavity axis $(x)$ and has a small extent in the $y$ direction [15]. To minimize the optical loss, the electrode was brought in the proximity of the resonator along the $y$ direction and no significant degradation of the quality factor was observed. On the same chip, we measured electrically controlled resonators with quality factors as high as 17000 , similar to cavities without the metal electrode. The cavity studied in this Letter had a lower quality factor $(Q \sim 4000)$ because it was integrated with a grating structure that allows efficient resonant in- or outcoupling from the resonator, as discussed in [5].

The photonic crystal was fabricated in a GaAs membrane [4] located on top of an $\mathrm{Al}_{0.8} \mathrm{Ga}_{0.2} \mathrm{As}$ sacrificial layer that was removed by wet etching in $\mathrm{HF}(6 \%)$ to create the suspended structure. Before the wet etch, metal contacts were defined using electron beam lithography followed by thermal evaporation and metal lift-off in acetone, which completely removed any residual electron beam resist. The measurements were done at cryogenic temperatures using a cross-polarized optical setup as shown in Fig. 1(b). First, a photoluminescence (PL) measurement was performed to identify a strongly coupled QD. The signature of strong coupling is the vacuum Rabi splitting, observed [Fig. 1(c)] as an avoided crossing of the eigenstates of the system when the quantum dot is tuned into resonance with the cavity [7]. From the PL spectra, one could identify two quantum dots with frequencies close to the cavity resonance, labeled as QD1 and QD2 in Fig. 2(a). Only QD1 showed the avoided crossing, thus indicating strong coupling. All of the measurements reported in this Letter were done using QD1, but the signature of QD2 was still visible in some of the data sets. For clarity, QD1 was marked with a red arrow in some of the figures. The experimental data indicated a cavity quality factor $Q \sim 4000$, corresponding to a field decay rate $\kappa / 2 \pi \sim 40 \mathrm{GHz}$ and a quantum dot cavity coupling strength $g / 2 \pi \sim 20 \mathrm{GHz}$. Since $g \geq \kappa / 2$ and $g \gg \gamma$ ( quantum dot spontaneous emission rate $\gamma / 2 \pi$ on the order of $0.1 \mathrm{GHz}$ ), the system operated on the onset of the strong coupling regime.

The vacuum Rabi splitting was also observed in the transmission function of the resonator, as measured using a cross-polarized reflectivity measurement [Fig. 2(b)] [4]. Two types of resonant probing were used in this experiment. In one case, a continuous wave $(\mathrm{cw})$ laser beam was scanned through the cavity resonance and the output was monitored with a photodetector. This measurement is re-


FIG. 2 (color online). (a) Photoluminescence spectra as a function of increasing control voltage $(V)$ from $0 \mathrm{~V}$ to $10 \mathrm{~V}$. At $0 \mathrm{~V}$ the quantum dot was tuned on resonance with the cavity $(T=48 \mathrm{~K})$. The PL intensity decreases and the QD redshifts for $V>8 \mathrm{~V}$. The shift is much smaller than the one observed in broadband reflectivity because of the screening induced by free carriers. (b) Broadband reflectivity spectra while changing $V$ from $0 \mathrm{~V}$ to $10 \mathrm{~V}(T=48 \mathrm{~K})$. The QD redshifts for $V>4 \mathrm{~V}$.

ferred to as "cw reflectivity." In the second case, a broadband light source was coupled into the resonator and the entire reflectivity spectrum was monitored on a spectrometer. This measurement is referred to as "broadband reflectivity."

With the quantum dot and the cavity brought into resonance (temperature set to $T=48 \mathrm{~K}$ ), the effect of the electric field was first studied in PL by changing the bias voltage from $0 \mathrm{~V}$ to $10 \mathrm{~V}$. As the bias approached $\sim 10 \mathrm{~V}$, the total PL intensity decreased and the quantum dot showed a redshift of only $\sim 0.03 \mathrm{~nm}(0.04 \mathrm{meV})$ as shown in Fig. 2(a). The shift in the QD resonance was due to the QCSE, and the reduction in the PL intensity was caused by the carriers being swept away before recombining in the QD. The Stark shift and the PL reduction were only observed when using low powers of the excitation laser (tuned at $875 \mathrm{~nm}$ ). By increasing the intensity of the laser, more carriers were excited in the cavity and thus screened the electric field.

To test the effect of the electric field under resonant probing, the system was measured using broadband reflectivity. A superluminescent diode with broad emission around $935 \mathrm{~nm}$ was used as the light source, thus minimizing free carrier generation because of its low spectral power density. The broadband reflectivity spectra shown in Fig. 2(b)indicate two local minima (marked by the red arrow for the strongly coupled dot and green arrow for the weakly coupled dot), thus confirming that two quantum dots were coupled to the cavity. The effect of the bias voltage on the QD wavelength was more pronounced than in the PL measurement. The Stark shift could be observed for bias voltages larger than $V \sim 4 \mathrm{~V}$, and for voltages exceeding $V \sim 7 \mathrm{~V}$ the quantum dot was completely detuned from the cavity. As seen from Fig. 2(b), by 
applying the electric field the transmission at the cavity resonance is switched from a local minimum to a local maximum.

The dependence of the quantum dot Stark shift with the applied bias voltage is shown in Fig. 3(a). The quantum dot frequency was determined by fitting the spectra in Fig. 2(b), as described in Ref. [4]. The Stark shift is only observed for voltages larger than $V \sim 4 \mathrm{~V}$, which corresponds to the depletion layer extending to the location of the quantum dot. The magnitude of the electric field in the center of the cavity was inferred by modeling the Schottky contact. The size of the depletion layer $\left[x_{d}(V)\right]$ and the electric field in the cavity $[F(V)]$ are given by $x_{d}=$ $\sqrt{2 \epsilon_{0} \epsilon_{\mathrm{GaAs}}(V-\phi) /\left(e N_{d}\right)} \quad$ and $\quad F=-e N_{d}\left(x_{d}-\right.$ $\Delta x) H\left(x_{d}-\Delta x\right) /\left(\epsilon_{0} \epsilon_{\mathrm{GaAs}}\right)$. Here, $\Delta x=750 \mathrm{~nm}$ is the distance between the electrode and the center of the cavity, $N_{d}=9 \times 10^{15} / \mathrm{cm}^{3}$ is the doping concentration, $\phi=$ $0.36 \mathrm{~V}$ is the potential barrier of the Schottky contact, $e$ is the electron charge, $\epsilon_{\mathrm{GaAs}}=12.9$ is the dielectric constant of GaAs at low temperatures, $\epsilon_{0}$ is the electrical permittivity of vacuum, and $H(x)$ is the unit-step function. The effect of the surface states was not considered when estimating the electric field. The dependence of the energy shift with electric field is shown in Fig. 3(b). The shift was
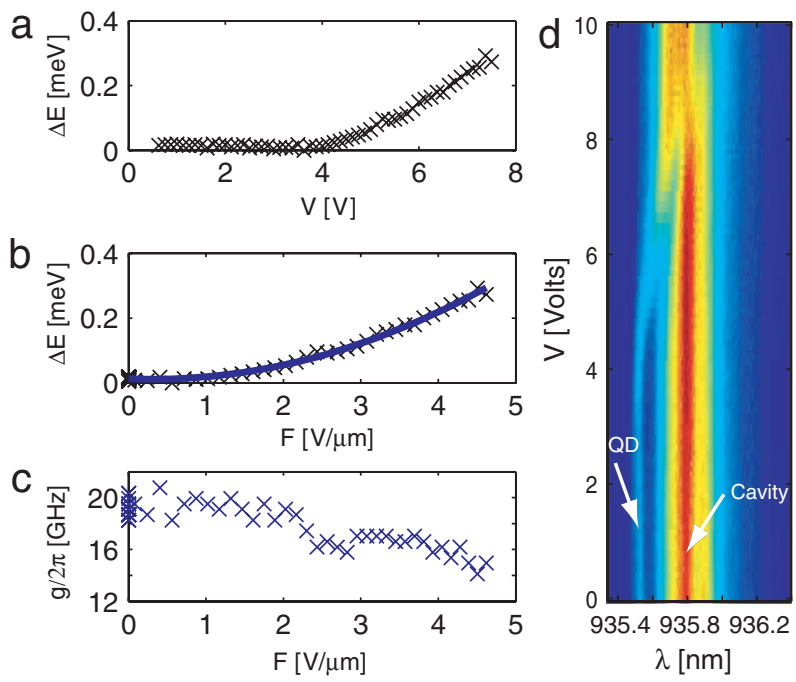

FIG. 3 (color online). Change in QD frequency and coupling strength $g$ with bias voltage. (a) Stark shift of the quantum dot with applied bias. (b) Experimental data and fit indicating the quadratic dependence of the quantum dot shift with electric field. (c) Dependence of the cavity/QD coupling $g$ with applied voltage, as inferred from the fit to experimental data (d) Broadband reflectivity taken at $T=46 \mathrm{~K}$ such that the QD was resonant with the cavity at high electric fields. As the voltage approaches $8 \mathrm{~V}$, the signature of the quantum dot in the spectrum vanished, most probably due to the loss in the quantum dot confinement due to high electric field. More information about the nature of this effect could be inferred by a QD spectroscopy method where the energy of all of the QD orbitals are monitored while varying the field. quadratic in electric field, since the perturbation of the energy levels due to electric field is a second order effect. The data were fit using [14] $\Delta E=p F-\beta F^{2}$ with $\beta=$ $-0.015 \mathrm{meV} \mu \mathrm{m}^{2} / \mathrm{V}^{2}=-2.4 \times 10^{-36} \mathrm{~J} /(\mathrm{V} / \mathrm{m})^{2} \quad$ and $p=-0.009 \mathrm{meV} \mu \mathrm{m} / \mathrm{V}=-1.4 \times 10^{-30} \mathrm{Cm}$. These values are within an order of magnitude but lower than previously measured by other research groups $[14,16,17]$. We believe that the lower value that we estimate is caused by the QD not being located exactly in the middle of the cavity, as we assumed.

The confining potential of the quantum dot could be perturbed by the influence of the electric field. For the data set shown in Fig. 2(b) (taken at $T=48 \mathrm{~K}$ ), the QD became completely off resonant with the cavity for $V>$ $7 \mathrm{~V}$ so the reflectivity spectrum at high electric field yielded little information about the quantum dot. For a better investigation of the QD behavior at large electric fields, another data set was taken at $T=46 \mathrm{~K}$ [Fig. 3(d)] such that the QD was resonant with the cavity for $V>7 \mathrm{~V}$. Under bias voltage, the electron and hole wave functions were deformed and pulled in opposite directions, thus reducing their overlap. One consequence is diminished QD oscillator strength. The overall result is in a reduction of the cavity/QD coupling $g$. The fits to the data showed that $g / 2 \pi$ decreased from $\sim 20 \mathrm{GHz}$ to $\sim 15 \mathrm{GHz}$ when a bias of $\sim 7 \mathrm{~V}$ was applied. For $V>8 \mathrm{~V}$, the influence of the electric field was strong enough to completely erase the signature of the quantum dot from the broadband reflectivity spectrum. This could be either due to reduced $g$ or to the high tunneling rate of the electron-hole pairs out of the quantum dot placed in the electric field.

The cw reflectivity spectra for different values of the bias voltage are shown in Fig. 4(a). The Stark shift was still present but its magnitude was smaller compared to the broadband reflectivity measurement $(0.04 \mathrm{meV}$ for $V \sim$ $10 \mathrm{~V}$ ), most probably due to an increased number of carriers that screen the electric field. We believe that, unlike the broadband source, the $\mathrm{cw}$ laser was more efficient in generating excitons in the quantum dot because of
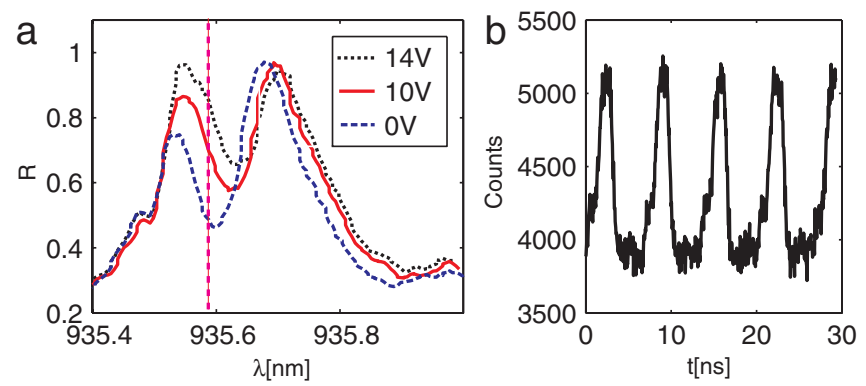

FIG. 4 (color online). Fast electrical switching of a continuous wave laser beam. (a) cw reflectivity for bias voltages of 0,10 , and $14 \mathrm{~V}$. During the time-domain switching experiment, the laser was set at the wavelength marked by the vertical dashed line and a $0-10 \mathrm{~V}$ signal was applied. (b) Switching of the coupled laser at the electrical QD driving frequency of $150 \mathrm{MHz}$. 
increased spectral power density and long coherence length. Because of the bias voltage, these electron-hole pairs could tunnel out of the quantum dot and become free carriers that screened the electric field [18]. Alternatively, since more than one quantum dot was present in the cavity, the screening could also be caused by excitons created in the neighboring off resonant quantum dots. It has already been shown that these excitons could be created through the off resonant energy transfer between the photonic-crystal resonator and the coupled quantum dots $[5,8]$. The screening of the electric field became more pronounced with increasing laser intensity, thus affecting the device performance. The effect of the QD on the transmission function of the resonator can be observed for coupled probe powers as high as tens of $\mathrm{nW}$ (as previously shown in [4]), but manipulation of the QD via QCSE at those probe power levels still needs to be demonstrated and may be limited by electric field screening. The cw reflectivity spectra in Fig. 4(a) indicated that an on/off switching ratio of $\sim 1.5: 1$ was achievable with this system when driven between 0 and $10 \mathrm{~V}$ (on/off ratio of 2:1 achievable for $0-14 \mathrm{~V}$ driving). Although on:off ratios of 100:1 are theoretically expected with this system, the experimentally observed on/off ratio was limited by the properties of the quantum dot, especially operation at the onset of the strong coupling regime, decoherence [5], and QD blinking [19].

The time-domain measurement was performed by setting the probe laser at the QD frequency [marked by the vertical dashed line in Fig. 4(a)] and by controlling the voltage using a function generator. The modulated output was monitored using a single-photon counting module (SPCM) and a dual channel picosecond time analyzer (PTA) synchronized to the function generator. To minimize the amount of screening due to coherently excited carriers, the probe laser power was set to $\sim 10 \mathrm{pW}$. The switching behavior at $150 \mathrm{MHz}$ is shown in Fig. 4(b), with an on/off ratio of $\sim 1.3: 1$. This is smaller than the expected $\sim 1.5: 1$ because of the $3 \mathrm{~dB}$ cutoff in the transmission line at $100 \mathrm{MHz}$. An on/off ratio of 1.45:1 was observed when driving the system at $80 \mathrm{MHz}$, close to the value expected from the dc measurement.

The performance of the proof of concept device reported in this Letter is limited by the experimental setup and the nonideality of the strongly coupled system. All-optical measurements on similar devices showed that speeds up to $10 \mathrm{GHz}$ could be achieved with this type of system [5]. With improved engineering, similar speeds should be achievable in electrical operation. Theoretically, when operating with $g, \kappa \gg \gamma$ (i.e., strong coupling regime or high Purcell factor regime) as is the case for quantum dots in photonic crystals, the maximum bandwidth is limited to $\min (g / \pi, \kappa / \pi)$ in the strong coupling regime and $g^{2} /(\pi \kappa)$ in the weak coupling regime. Regarding the energy required to shift the QD, it is fundamentally limited by the energy density of the electric field required to shift the quantum dot inside the active volume. Considering an active volume the size of the resonator $\left(V_{a} \sim 1 \mu \mathrm{m} \times\right.$ $1 \mu \mathrm{m} \times 200 \mathrm{~nm})$ and an electric field $F \sim 5 \times$ $10^{4} \mathrm{~V} / \mathrm{cm}$, this translates into a switching energy of $\sim 1 f J$, much lower than state of the art devices [20-24]. Confining the electric field over such a small volume is not trivial, but suitable technological solutions may be found in the future.

In conclusion, we demonstrated dynamic control of QCSE in a QD strongly coupled to a photonic-crystal cavity. We reported electro-optic switching up to $150 \mathrm{MHz}$ with an on:off ratio of 1.3:1 and discussed the prospects for improving the device performance. This type of device can be integrated in an on-chip optical or quantum network [2] and will be an essential building block for future optoelectronic devices for classical and quantum information processing devices operating at ultralow energies, where fine and fast tuning of the quantum dot resonance is required.

Financial support was provided by the Presidential Early Career Grant, the DARPA Young Faculty Grant, and the Army Research Office. Part of the work was performed at the Stanford Nanofabrication Facility of NNIN supported by the National Science Foundation. A. M. was also supported by the Stanford Graduate program (Texas Instruments). The authors thank Professor David Miller for useful discussion.

*andrei.faraon@hp.com

[1] S. Noda, J. Lightwave Technol. 24, 4554 (2006).

[2] A. Faraon et al., Opt. Express 16, 12154 (2008).

[3] S. Noda et al., Nat. Photon. 1, 449 (2007).

[4] D. Englund et al., Nature (London) 450, 857 (2007).

[5] D. Englund et al., arXiv:0902.2428v2.

[6] D. Englund et al., Opt. Express 17, 18651 (2009).

[7] T. Yoshie et al., Nature (London) 432, 200 (2004).

[8] K. Hennessy et al., Nature (London) 445, 896 (2007).

[9] D. A. B. Miller et al., Phys. Rev. Lett. 53, 2173 (1984).

[10] D. A. B. Miller, Proc. IEEE 97, 1166 (2009).

[11] Y. Akahane et al., Nature (London) 425, 944 (2003).

[12] A. Högele et al., Phys. Rev. Lett. 93, 217401 (2004).

[13] A. Laucht et al., New J. Phys. 11, 023034 (2009).

[14] B. D. Gerardot et al., Appl. Phys. Lett. 90, 041101 (2007).

[15] A. Faraon et al., Appl. Phys. Lett. 90, 073102 (2007).

[16] V. Stavarache et al., Appl. Phys. Lett. 89, 123105 (2006).

[17] X. Xu et al., New J. Phys. 10, 053036 (2008).

[18] W. Heller et al., Phys. Rev. B 57, 6270 (1998).

[19] A. Faraon et al., Nature Phys. 4, 859 (2008).

[20] Q. Xu et al., Opt. Express 16, 4309 (2008).

[21] J. Liu et al., Nat. Photon. 2, 433 (2008).

[22] Q. Xu et al., Opt. Express 15, 430 (2007).

[23] A. Liu et al., Nature (London) 427, 615 (2004).

[24] X. Chen et al., Opt. Lett. 34, 602 (2009). 